\title{
Coherent Population Trapping in Two-Electron Three-Level Systems with Aligned Spins
}

\author{
Jordi Mompart, ${ }^{1}$ Ramon Corbalan, ${ }^{1}$ and Luis Roso ${ }^{1,2}$ \\ ${ }^{1}$ Departament de Física, Universitat Autònoma de Barcelona, E-08193 Bellaterra, Spain \\ ${ }^{2}$ Departamento de Fisica Aplicada, Universidad de Salamanca, E-37008 Salamanca, Spain
}

(Received 25 July 2001; published 21 December 2001)

\begin{abstract}
The possibility of coherent population trapping in two electron states with aligned spins (orthosystem) is evidenced. From the analysis of a three-level atomic system containing two electrons, and driven by the two laser fields needed for coherent population trapping, a conceptually new kind of dark state appears. The properties of this trapping are physically interpreted in terms of a dark hole, instead of a dark two-electron state. This technique, among many other applications, offers the possibility of measuring, with subnatural resolution, some superposition-state matrix elements of the electron-electron correlation that due to their time dependent nature are inaccessible by standard measuring procedures.
\end{abstract}

It has been well known since the late seventies [1-3] that the application of two continuous wave laser fields to a three-level atomic $\Lambda$ system leads to the preparation of the atom in a coherent superposition state named the dark state, which is stable against absorption from the applied fields. This phenomenon is known as coherent population trapping, to indicate the presence of a coherent superposition of atomic states and the stability of the population in this state. Of course, there is another superposition of atomic states, orthogonal to the dark state and coupled to the applied fields, which is named bright state. The dark and bright states have been fruitfully employed in thousands of different situations [4]. Related concepts, such as electromagnetically induced transparency, amplification without inversion, or lasing without inversion, have been also introduced over the past few years [5,6].

Most of the related experiments deal with singleelectron atoms, or single active electron situations, where an electron is coherently forced to a superposition state. In a two-electron system, the situation is different because of the electron-electron interaction and particularly because of the Pauli exclusion principle. In this case, the dynamics of one of the electrons will strongly influence the dynamics of the second one. Of course, to show this effect, one needs to work with electrons having parallel spins. If spins were antiparallel, then both of them could be allowed in the same atomic (spatial) state. There are some studies that use related properties of the two-electron systems in case of antiparallel spins [7], in different contexts, namely, double-core resonance.

In the present Letter, we restrict ourselves to the case of a two-electron orthosystem [8], and therefore the Pauli exclusion principle acts on the spatial part of the wave functions. This prevents both electrons from being in the same spatial state. For the case of coherent population trapping this has rich consequences of a very fundamental nature that have never been considered before. We present the general case; we discuss some possibilities for experimental realization of such a system, and we propose the use of coherent population trapping to measure some timedependent electron-electron interaction matrix elements.

Let us consider a typical V-configuration of the three single-electron states, as indicated in Fig. 1, labeled $|a\rangle$, $|b\rangle$, and $|c\rangle$, with energies $\hbar \omega_{a}, \hbar \omega_{b}$, and $\hbar \omega_{c}$, respectively. If two of these states are populated, we can build up antisymmetrized two-electron states. Since two electrons with aligned spins are considered, the spin term is symmetric and the antisymmetry of the total wave function comes from the spatial part of the wave function.

The three-level system is formed by two adjacent dipole transitions sharing a common state $|c\rangle$. The dipole coupling to the laser fields, $H_{\text {dip }}$, is given by $\left\langle a\left|H_{\text {dip }}\right| c\right\rangle=$ $\hbar \alpha e^{-i \omega_{\alpha} t}$, and $\left\langle b\left|H_{\text {dip }}\right| c\right\rangle=\hbar \beta e^{-i \omega_{\beta} t}$, where $\alpha$ and $\beta$ are the Rabi frequencies of the two laser fields, and $\omega_{\alpha}$ and $\omega_{\beta}$ represent their frequencies. We assume that all three single-electron states involved have definite parity, with $|a\rangle$ and $|b\rangle$ having the same parity and $|c\rangle$ having the opposite one. Therefore, $\left\langle a\left|H_{\text {dip }}\right| a\right\rangle=\left\langle b\left|H_{\text {dip }}\right| b\right\rangle=$ $\left\langle c\left|H_{\text {dip }}\right| c\right\rangle=0$, and also $\left\langle a\left|H_{\text {dip }}\right| b\right\rangle=0$. We introduce the rotating wave approximation [2,9], just keeping slow oscillations at frequencies comparable to the detunings, $\Delta_{\alpha}=\omega_{\alpha}-\left(\omega_{a}-\omega_{c}\right)$, and $\Delta_{\beta}=\omega_{\beta}-\left(\omega_{b}-\omega_{c}\right)$. We introduce the relaxation coefficient $\gamma_{a c}$, indicating the rate of decay of the population from state $|a\rangle$ (the uppermost level) to $|c\rangle$, and the relaxation coefficient $\gamma_{b c}$, indicating the rate of decay of the population from $|b\rangle$ to $|c\rangle$. In the dipole approximation, $\gamma_{b a}=\gamma_{a b}=0$.

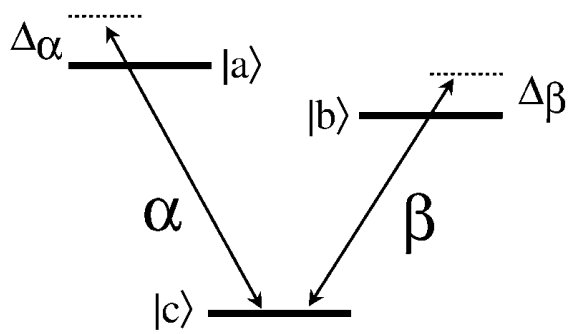

FIG. 1. Single-electron states, $|a\rangle,|b\rangle$, and $|c\rangle$, in a V configuration. 
Let us consider now that two electrons (in the same spin state) are forced to be inside this three-level system. The two-electron states are indicated by $|i, j\rangle=|i\rangle \otimes|j\rangle$, with $i, j=a, b, c$. Antisymmetrized states are $|A\rangle=$ $(1 / \sqrt{2})(|c, b\rangle-|b, c\rangle), \quad|B\rangle=(1 / \sqrt{2})(|a, c\rangle-|c, a\rangle)$, and $|C\rangle=(1 / \sqrt{2})(|a, b\rangle-|b, a\rangle)$. Without electronelectron interaction, the energies are given by $\hbar$ times $\omega_{A}=\omega_{b}+\omega_{c}$, for state $|A\rangle, \omega_{B}=\omega_{a}+\omega_{c}$, for state $|B\rangle$, and $\omega_{C}=\omega_{a}+\omega_{b}$, for state $|C\rangle$.

Parity of the two-electron states is directly related to parity of the single-electron states. Two-electron states will thus keep a well-defined parity. $|A\rangle$ and $|B\rangle$ are of the same parity and $|C\rangle$ of the opposite one. The two-electron state dipole matrix elements will be $\left\langle C\left|H_{\text {dip }}\right| A\right\rangle=\hbar \alpha e^{-i \omega_{\alpha} t}$, and $\left\langle C\left|H_{\text {dip }}\right| B\right\rangle=\hbar \beta e^{-i \omega_{\beta} t}$; all other couplings are zero because of parity considerations, $\quad\left\langle A\left|H_{\text {dip }}\right| A\right\rangle=\left\langle B\left|H_{\text {dip }}\right| B\right\rangle=\left\langle C\left|H_{\text {dip }}\right| C\right\rangle=$ $\left\langle A\left|H_{\text {dip }}\right| B\right\rangle=0$. These two-electron states determine now a $\Lambda$ configuration, see Fig. 2, while the one-electron states $|a\rangle,|b\rangle$, and $|c\rangle$ formed a $\mathrm{V}$ configuration.

The density matrix for the two-electron three-level system is defined by $(\rho)_{I J}=|I\rangle\langle J|$, with $I, J=A, B, C$. Still, without considering the electron-electron interaction, the dynamical equations for the two-electron density matrix are

$$
\begin{aligned}
\frac{d}{d t} \rho_{A A}= & i\left[\rho_{A C} \alpha-\rho_{A C}^{*} \alpha^{*}\right]+\gamma_{C A} \rho_{C C}, \\
\frac{d}{d t} \rho_{B B}= & i\left[\rho_{B C} \beta-\rho_{B C}^{*} \beta^{*}\right]+\gamma_{C B} \rho_{C C} \\
\frac{d}{d t} \rho_{C C}= & i\left[-\rho_{A C} \alpha+\rho_{A C}^{*} \alpha^{*}-\rho_{B C} \beta+\rho_{B C}^{*} \beta^{*}\right] \\
& -\left(\gamma_{C A}+\gamma_{C B}\right) \rho_{C C}, \\
\frac{d}{d t} \rho_{A B}= & i\left[-\rho_{A C}\left(\Delta_{\alpha}-\Delta_{\beta}\right)+\rho_{A C} \beta-\rho_{B C}^{*} \alpha^{*}\right] \\
& -\Gamma_{A B} \rho_{A B}, \\
\frac{d}{d t} \rho_{A C}= & i\left[-\rho_{A C} \Delta_{\alpha}+\alpha^{*}\left(\rho_{A A}-\rho_{C C}\right)+\rho_{A B} \beta^{*}\right] \\
& -\Gamma_{A C} \rho_{A C}, \\
\frac{d}{d t} \rho_{B C}= & i\left[-\rho_{B C} \Delta_{\beta}+\beta^{*}\left(\rho_{B B}-\rho_{C C}\right)+\rho_{A B}^{*} \alpha^{*}\right] \\
& -\Gamma_{B C} \rho_{B C} .
\end{aligned}
$$

The relaxations corresponding to single-electron processes, $\gamma_{C A}=\gamma_{a c}, \quad \gamma_{C B}=\gamma_{b c}$, indicate an electron falling from $|a\rangle$ to $|c\rangle$, and from $|b\rangle$ to $|c\rangle$, respectively. Dipole decay between states $|a\rangle$ and $|b\rangle$ is forbidden by parity: $\gamma_{B A}=\gamma_{a b}=0$, and $\gamma_{A B}=$ $\gamma_{b a}=0$. The two-electron coherences will relax according to $2 \Gamma_{B A}=\gamma_{B A}+\gamma_{A B}=0,2 \Gamma_{A C}=\gamma_{C A}+\gamma_{C B}=$ $\gamma_{a c}+\gamma_{b c}$, and $2 \Gamma_{B C}=\gamma_{C A}+\gamma_{C B}=\gamma_{a c}+\gamma_{b c}$. Simultaneous two-electron relaxation mechanisms are forbidden in the electric dipole approximation.

Following standard ideas of solid state physics, we have two electrons to fill three states, $|a\rangle$, $|b\rangle$, and $|c\rangle$. So

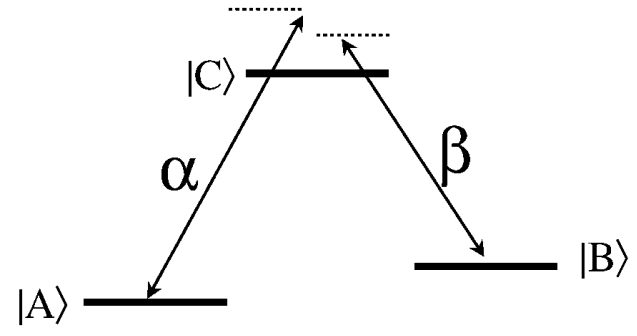

FIG. 2. Representation of the two-electron states, $|A\rangle,|B\rangle$, and $|C\rangle$, automatically arranged in a $\Lambda$ configuration.

there are two occupied states plus an empty one, the hole. The system is thus characterized by the position of the hole. The two-electron state $|C\rangle$, for example, involves one electron at $|a\rangle$ and the second electron at $|b\rangle$, leaving $|c\rangle$ empty.

It is perfectly well established in one-electron three-level theory [1-3] that the two lower states of a $\Lambda$ scheme form very peculiar coherent superposition states. In principle, these superposition states should be also possible in the two upper states of a single-electron V-scheme system. In this case, the result is that a state $|d\rangle$ uncoupled to the laser fields and another state $|e\rangle$ coupled to the laser fields appear. Nevertheless these single-electron states are not precisely the typical dark and bright states because they correspond to the two upper states of the $\mathrm{V}$ configuration and relax very fast. In the particular case that the Rabi frequencies of both transitions are equal, $\alpha=\beta$, and the detunings verify $\Delta_{\alpha}=\Delta_{\beta}$, then the expressions of the dark, $|d\rangle$, and the bright, $|e\rangle$, states are extremely simple and symmetrical, $|d\rangle=(1 / \sqrt{2})(|a\rangle-|b\rangle)$, and $|e\rangle=(1 / \sqrt{2})(|a\rangle+|b\rangle)$.

We can repeat the procedure to obtain the dark states now using two-electron states. The result is that a dark state $|D\rangle$ and a bright state $|E\rangle$ appear. In the particular case of equal Rabi frequencies, $\alpha=\beta$, and equal detunings $\Delta_{\alpha}=\Delta_{\beta}$, then the dark state is $|D\rangle=(1 / \sqrt{2})(|A\rangle-|B\rangle)$ and the bright state is $|E\rangle=$ $(1 / \sqrt{2})(|A\rangle+|B\rangle)$. Now the dark state $|D\rangle$ involves one electron in a superposition of the upper states $|a\rangle$ and $|b\rangle$, while the other electron lies in the lowest energy state $|c\rangle$. Therefore a hole appears in the empty upper state. Relaxation does not play a role now because the lower state is filled with an electron and the Pauli exclusion principle does not allow a second one in the same state. Therefore this dark state has the appearance of a hole that is moving between the two upper single-electron states. To illustrate this we have included a new scheme of the single-electron states. Figure $3 \mathrm{a}$ corresponds to only one electron (grey circle) in the system. Under the appropriated conditions, the electron can be placed in a state $|d\rangle$ that is not coupled to the fields. This state, however, can decay. In the case of two electrons, Fig. 3b, there is one electron at the lower state that prevents the decay from the upper states. The state without an electron, the hole, is trapped and stable. Therefore, we have now $a$ 
(a)

(b)

$|\mathrm{d}\rangle$

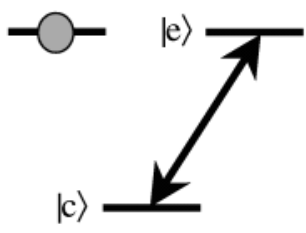

$|\mathrm{d}\rangle$

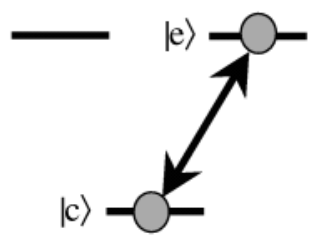

FIG. 3. Representation of a three-level single-electron system. If only one electron is considered (a) a uncoupled state $|d\rangle$ appears as a superposition of the two excited states. State $|d\rangle$ may decay to the lower state. If two electrons are considered (b) there is a hole that can be trapped in this uncoupled state. This hole cannot relax to any other state.

hole placed at a dark state. Notice finally that, since $|D\rangle$ is a two-particle state insensitive to spontaneous emission decay and inelastic collisions, it could have interesting applications for quantum information processing.

We have thus far considered two-electron systems where the electron-electron interaction is not accounted for. Only the Pauli exclusion principle has been considered through the antisymmetrization of the spatial part of the wave function (spin part is always symmetrical in the considered orthoatom). The two-electron system can be understood in terms of a hole. Of course this agreement is thus far perfect because we have forgotten one particular feature, the electronelectron repulsion. Now it is time to consider these terms and see how they modify the presented results. The interaction Hamiltonian is $H_{e e}=e^{2} /\left|\mathbf{r}_{1}-\mathbf{r}_{2}\right|, \mathbf{r}_{1}$ and $\mathbf{r}_{2}$ being the positions of the electrons, then

$$
\begin{gathered}
\left\langle A\left|H_{e e}\right| A\right\rangle=\hbar \Delta_{A}, \\
\left\langle B\left|H_{e e}\right| B\right\rangle=\hbar \Delta_{B}, \\
\left\langle C\left|H_{e e}\right| C\right\rangle=\hbar \Delta_{C}, \\
\left\langle A\left|H_{e e}\right| C\right\rangle=0, \\
\left\langle B\left|H_{e e}\right| C\right\rangle=0, \\
\left\langle A\left|H_{e e}\right| B\right\rangle=\hbar \chi e^{-i \omega_{b} t} e^{i \omega_{a} t} .
\end{gathered}
$$

These electron-electron interaction terms can be grouped in two different families, the time-independent terms (that do not contain time oscillations at the energy difference) and the time-dependent terms (that do contain explicit time oscillations). Time-independent terms come from the diagonal matrix elements $\left\langle A\left|H_{e e}\right| A\right\rangle,\left\langle B\left|H_{e e}\right| B\right\rangle$, and $\left\langle C\left|H_{e e}\right| C\right\rangle$. The contributions $\left\langle c, b\left|H_{e e}\right| c, b\right\rangle$, $\left\langle c, a\left|H_{e e}\right| c, a\right\rangle$, and $\left\langle b, a\left|H_{e e}\right| b, a\right\rangle$ are Coulomb terms. The contributions $\left\langle c, b\left|H_{e e}\right| b, c\right\rangle,\left\langle c, a\left|H_{e e}\right| a, c\right\rangle$, and $\left\langle b, a\left|H_{e e}\right| a, b\right\rangle$ are exchange terms. For a system in a pure quantum state, only these two kinds of electron-electron terms are relevant. With the dynamical situation established via the interaction with the laser fields, electrons are in superposition states and, thus, new terms may appear that are time dependent. The term

$\left\langle A\left|H_{e e}\right| B\right\rangle$ is very particular because it involves three different one-electron states. It is the sum of two different contributions, $\left\langle c, b\left|H_{e e}\right| a, c\right\rangle$ and $\left\langle c, b\left|H_{e e}\right| c, a\right\rangle$. The cross term $\left\langle c, b\left|H_{e e}\right| a, c\right\rangle$ involves the two dipole-allowed transitions: $|a\rangle \leftrightarrow|c\rangle$ for one electron, and $|c\rangle \leftrightarrow|b\rangle$ for the other electron. Another cross term that appears is $\left\langle c, b\left|H_{e e}\right| c, a\right\rangle$; it is different from the first one because one of the electrons remains in the common state $|c\rangle$ while the other is in a $|a\rangle \leftrightarrow|b\rangle$ coherence. In any case, these two terms present a time oscillation at frequency $\omega_{a}-\omega_{b}$, so the resulting electron-electron matrix element can be written as $\left\langle A\left|H_{e e}\right| B\right\rangle=\hbar \chi e^{-i \omega_{b} t} e^{i \omega_{a} t}$. Finally, due to parity considerations, $\left\langle A\left|H_{e e}\right| C\right\rangle=\left\langle B\left|H_{e e}\right| C\right\rangle=0$ because $H_{e e}$ is an even operator.

Now we can introduce the $H_{e e}$ couplings in the time evolution of the density matrix. Taking the corrected energy of state $|A\rangle$ as the energy origin and introducing the rotating wave approximation, we obtain

$$
\begin{gathered}
\frac{d}{d t} \rho_{A A}=i\left[\rho_{A C} \alpha-\rho_{A C}^{*} \alpha^{*}\right]+\gamma_{C A} \rho_{C C} \\
+i\left[\rho_{A B} \chi^{*} e^{i \omega_{a} t} e^{-i \omega_{b} t}\right. \\
\left.-\rho_{A B}^{*} \chi e^{-i \omega_{a} t} e^{i \omega_{b} t}\right] \\
\frac{d}{d t} \rho_{B B}=i\left[\rho_{B C} \beta-\rho_{B C}^{*} \beta^{*}\right]+\gamma_{C B} \rho_{C C} \\
+i\left[\rho_{A B}^{*} \chi e^{-i \omega_{a} t} e^{i \omega_{b} t}\right. \\
\left.-\rho_{A B} \chi^{*} e^{i \omega_{a} t} e^{-i \omega_{b} t}\right] \\
\frac{d}{d t} \rho_{C C}=i\left[-\rho_{A C} \alpha+\rho_{A C}^{*} \alpha^{*}-\rho_{B C} \beta+\rho_{B C}^{*} \beta^{*}\right] \\
-\left(\gamma_{C A}+\gamma_{C B}\right) \rho_{C C}, \\
\frac{d}{d t} \rho_{A B}=i\left[-\rho_{A C}\left(\Delta_{\alpha}-\Delta_{\beta}\right)+\rho_{A C} \beta-\rho_{B C}^{*} \alpha^{*}\right] \\
-\Gamma_{A B} \rho_{A B} \\
+i\left[\left(\rho_{A A}-\rho_{B B}\right) \chi e^{-i \omega_{a} t} e^{i \omega_{b} t}\right. \\
\left.+\rho_{A B}\left(\Delta_{A}-\Delta_{B}\right)\right],
\end{gathered}
$$

$$
\begin{aligned}
\frac{d}{d t} \rho_{A C}= & i\left[-\rho_{A C} \Delta_{\alpha}+\alpha^{*}\left(\rho_{A A}-\rho_{C C}\right)+\rho_{A B} \beta^{*}\right] \\
& -\Gamma_{A C} \rho_{A C} \\
& +i\left[\rho_{A C}\left(\Delta_{C}-\Delta_{A}\right)-\rho_{B C} \chi e^{-i \omega_{\alpha} t} e^{t a t}\right],
\end{aligned}
$$

$$
\begin{aligned}
\frac{d}{d t} \rho_{B C}= & i\left[-\rho_{B C} \Delta_{\beta}+\beta^{*}\left(\rho_{B B}-\rho_{C C}\right)+\rho_{A B}^{*} \alpha^{*}\right] \\
& -\Gamma_{B C} \rho_{B C} \\
& +i\left[\rho_{B C}\left(\Delta_{C}-\Delta_{A}\right)-\rho_{A C} \chi^{*} e^{i \omega_{\alpha} t} e^{-i \omega_{\beta} t}\right] .
\end{aligned}
$$




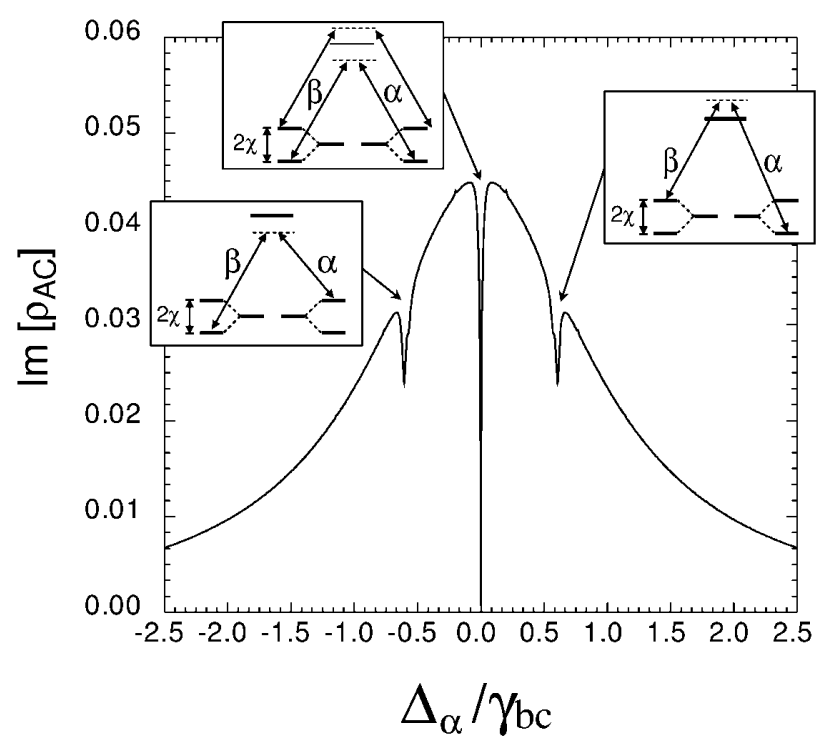

FIG. 4. Spectra of the dark state resonance and its two satellites induced by the crossed correlation terms. The figure represents the imaginary part of the $A C$ coherence versus the detuning $\Delta_{\alpha} / \gamma_{b c} . \gamma_{a c}=\gamma_{b c}, \alpha=\beta=0.1 \gamma_{b c}, \Delta_{\beta}=0$, and $\chi=0.3 \gamma_{b c}$. Insets indicate the dressed-level couplings.

This set of equations provides a description of the dynamics of the three-level two-electron system, including time-dependent electron-electron correlations.

One clear example where coherent population trapping of this kind could be observed is calcium. Other alkaline-earth elements are also good candidates. In the orthoatom case the ground state is $n s n p$ ( $4 s 4 p$ for calcium). One excited state very interesting is the nрnp. If a $\sigma^{+}, \sigma^{-}$laser field is considered, then the three single-electron states involved will be $|a\rangle=\left|n p_{+}\right\rangle,|b\rangle=$ $\left|n p_{-}\right\rangle$, and $|c\rangle=|n s\rangle$. Obviously, the three-two electron states will become $|A\rangle=\left|n s, n p_{-}\right\rangle,|B\rangle=\left|n s, n p_{+}\right\rangle$, and $|C\rangle=\left|n p_{+}, n p_{-}\right\rangle$. In this particular case, $\left\langle A\left|H_{e e}\right| B\right\rangle=0$ due to the angular momentum addition laws [10]. Because $n s n p_{+}$and $n s n p_{-}$correspond to the ground state of the orthoatom, they are relatively stable. In that case a clear dark state can be formed with all the characteristics of the coherent population trapping, except that the trapping affects the hole that is in a coherent superposition of the two excited single-electron states. This structure is also present in ortholithium (three aligned spins) involving the $1 s 2 s 2 p$ and $1 s 2 p 2 p$ states, as well as in other systems.

In the opposite case, for atoms or molecules where the $\left\langle A\left|H_{e e}\right| B\right\rangle$ matrix element is nonzero, we predict a similar dark resonance but shifted by an amount equal to the time average of the $\left\langle A\left|H_{e e}\right| B\right\rangle$ coupling. This suggests a new method to measure electron-electron correlations with subnatural resolution. To illustrate this, Fig. 4 has been included. This figure represents the spectra of the dark state resonance and its two satellites induced by the crossed correlation terms of the electron-electron interaction. It is a plot of the imaginary part of the $|A\rangle \leftrightarrow|C\rangle$ coherence versus the $\Delta_{\alpha}$ detuning, and corresponds to $\gamma_{a c}=\gamma_{b c}$, $\alpha=0.1 \gamma_{b c}, \beta=0.1 \gamma_{b c}, \Delta_{\beta}=0$, and $\chi=0.3 \gamma_{b c}$. For simplicity, all parameters have been referred to as $\gamma_{b c}$. Coherent population trapping appears at the center $\Delta_{\alpha}=0$, as expected. Moreover, two satellite holes appear at $\pm 2 \chi$. They are due to the splitting of the levels induced by the time-dependent matrix element and to its coupling to the laser field. The dressed transitions responsible for those peaks are depicted at the insets of Fig. 4.

In conclusion, we have analyzed the properties of a three-level two-electron system. The presence of a coherence between the atomic two-electron states leads to coherent population trapping similar to the one found in one-electron systems. However, this two-electron trapping presents some new and interesting features. Particularly remarkable is the presence of a hole (the empty state) trapped in a superposition of the upper states. In the case where the two lower states are coupled by the electronelectron potential, we propose a direct subnatural measure of these coupling coefficients. Finally, our approach allows to create stable superposition states at will of two fermionic undistinguishable particles which could be very useful to create entangled states for quantum information processing.

Partial support by the Spanish DGESIC (Grants No. PB98-0935-C03-03 and No. PB98-0268), by the Direcció Catalana de Recerca (Contract No. 1999SGR00096), and by the Junta de Castilla y León (Grant No. SA044/01) is acknowledged. We thank G. Orriols and V. Ahufinger for critical reading of the manuscript.

[1] G. Alzetta, A. Gozzini, L. Moi, and G. Orriols, Nuovo Cimento Soc. Ital. Fis. 36B, 5 (1976); E. Arimondo and G. Orriols, Lett. Nuovo Cimento 17, 333 (1976); G. Alzetta, L. Moi, and G. Orriols, Nuovo Cimento Soc. Ital. Fis. 52B, 205 (1979).

[2] G. Orriols, Nuovo Cimento Soc. Ital. Fis. 53B, 1 (1979).

[3] H. R. Gray, R. M. Whitley, and C. R. Stroud, Jr., Opt. Lett. 3, 218 (1978).

[4] E. Arimondo, in Progress in Optics, edited by E. Wolf (Elsevier, Amsterdam, 1996), Vol. 35, p. 257.

[5] S. E. Harris, Phys. Today 50, 36 (1997).

[6] J. Mompart and R. Corbalán, J. Opt. B 2, R7 (2000).

[7] R. Grobe, S. L. Haan, and J. H. Eberly, Phys. Rev. A 54, 1516 (1996).

[8] For analogy with the standard terminology with helium [orthohelium for the atom with parallel spins (triplet) and parahelium for the atom with antiparallel spins (singlet)], we will refer to orthoatom and paraatom.

[9] R. W. Boyd, Nonlinear Optics (Academic, New York, 1992).

[10] A. R. Edmons, Angular Momentum in Quantum Mechanics (Princeton University Press, Princeton, NJ, 1974). 